Meta

Journal des traducteurs

Translators' Journal

\title{
Pour la création d'une terminologie scientifique et technique en malgache
}

\section{Raphaël Rajaspera}

Volume 41, numéro 3, septembre 1996

URI : https://id.erudit.org/iderudit/003908ar

DOI : https://doi.org/10.7202/003908ar

Aller au sommaire du numéro

Éditeur(s)

Les Presses de l'Université de Montréal

ISSN

0026-0452 (imprimé)

1492-1421 (numérique)

Découvrir la revue

Citer cet article

Rajaspera, R. (1996). Pour la création d'une terminologie scientifique et technique en malgache. Meta, 41(3), 435-458. https://doi.org/10.7202/003908ar
Résumé de l'article

Cet article étudie les possibilités et les limites d'une malgachisation. L'auteur présente les divers procédés de création terminologique utilisés dans son pays et discute des problèmes de diffusion de cette terminologie. Il expose ensuite l'état présent du travail terminologique à Madagascar et les résultats à l'aide d'un lexique français-malgache des termes usités en cartographie. 


\title{
POUR LA CRÉATION D'UNE TERMINOLOGIE SCIENTIFIQUE ET TECHNIQUE EN MALGACHE
}

RAPHAËL RAJASPERA

Université d'Antananarivo, Antananarivo, Madagascar

\begin{abstract}
Résumé
Cet article étudie les possibilités et les limites d'une malgachisation. L'auteur présente les divers procédés de création terminologique utilisés dans son pays et discute des problèmes de diffusion de cette terminologie. Il expose ensuite l'état présent du travail terminologique à Madagascar et les résultats à l'aide d'un lexique français-malgache des termes usités en cartographie.
\end{abstract}

\begin{abstract}
This article deals with the limits and scope of "malgachisation". It gives an overview of current terminology research in Madagascar; discusses various new-word formation patterns as well as the problem of terminology, and concludes with a French-Malagasy lexicon of terms used in cartography.
\end{abstract}

\section{INTRODUCTION}

L'élaboration d'un lexique scientifique et technique n'est pas une entreprise nouvelle à Madagascar.

Déjà, vers la fin du xvme siècle, le botaniste espagnol Francisco Noroña avait rédigé un lexique malgache des noms de plantes ${ }^{1}$.

Depuis 1828 , la langue malgache disposait de tout un patrimoine juridique et linguistique dans le Code des 305 Articles, dont a hérité le droit malgache actuel. Font partie de ce patrimoine les formules, les sentences et les aphorismes de droit, de jurisprudence et de morale recueillis par Gustave Julien dans ses Institutions politiques et sociales de Madagascar ${ }^{2}$.

Dans la deuxième moitié du XIXe siècle, des missionnaires britanniques avaient entrepris de rédiger en langue malgache des ouvrages scientifiques qu'ils avaient traduits à partir de l'anglais : un manuel de botanique et un de géologie écrits en 1888 et en 1896 par le révérend Baron, un manuel de chirurgie générale par le docteur $O$. Thesen en 1894, une étude de zoologie par le révérend James Sibree en 1894 également, d'anatomie générale par le docteur Fox, assisté de ses disciples, en 1887, des manuels d'arithmétique et de mathématiques, etc. La terminologie utilisée pour les besoins de l'enseignement était souvent à base d'emprunts anglais malgachisés.

Pendant la période coloniale, qui a duré plus d'un demi-siècle, tout le travail de malgachisation de la terminologie avait été interrompu. Mais il a été repris à partir de 1960, au lendemain de l'indépendance de Madagascar, par diverses équipes de chercheurs malgaches et français.

Dans la situation actuelle, la base de la création terminologique est encore, dans l'ensemble, la traduction d'une langue européenne - le français - en malgache. 
Il s'agit désormais de voir, face au système relativement organisé des lexiques spécialisés en français, comment trouver des équivalents malgaches qui puissent répondre à diverses conditions: correspondre à un besoin, satisfaire à la clarté de la communication, respecter les particularités phonologiques et morpho-syntaxiques propres à la langue réceptrice et à sa structure sémantique.

Cela soulève un double problème: 1) celui des procédés de création terminologique, et 2) celui de la diffusion auprès du public. La question est alors de savoir comment s'effectue encore la création d'une terminologie technico-scientifique malgache, pour qui cette terminologie a été créée et quel usage en est fait.

\section{PROCÉDÉS DE CRÉATION D'UNE TERMINOLOGIE SCIENTIFIQUE ET TECHNIQUE EN MALGACHE}

Pour transmettre des concepts nouveaux et sémantiquement structurés dès la langue de départ, les créateurs d'une terminologie technique et scientifique malgache, devaient, à leur tour, élaborer un lexique nouveau aux structurations régulières. Ainsi, on a essayé, à l'image du français, de structurer la nouvelle langue scientifique et technique malgache sur le plan formel et sémantique. En cela, on a eu recours à différents types de néologismes apparus à une certaine période de la vie de la communauté linguistique malgache (1960-1990). Parmi eux, nous retiendrons: 1) les formations néologiques à caractère grammatical; 2) les formations néologiques à caractère lexical.

\section{LES FORMATIONS NÉOLOGIQUES À CARACTERE GRAMMATICAL}

Les formations néologiques à caractère grammatical prennent le plus souvent un aspect phonologique et un aspect morphologique.

LA NÉOLOGIE PHONOLOGIQUE

Parmi les procédés de création terminologique, la néologie phonologique joue encore un grand rôle aujourd'hui. Il s'agit, par le simple jeu de la combinatoire des phonèmes dans un paradigme autorisé par le système de la langue malgache, de former une substance signifiante nouvelle.

C'est ainsi que, dans le lexique de l'IREM, on a introduit des segments linguistiques de consonance nouvelle (ex. : ovaka «variance» et ovala «variable») à partir d'une base connue (ici ova «action de changer»).

La malgachisation, complète ou incomplète, des emprunts au français peut être considérée comme à l'origine de certains néologismes phonologiques. On relève, par exemple, les xénismes ôksida «oxyde», ohmmetatra «ohmmètre», ôsiloskôpy «oscillo-


l'intérieur du mot simple: les groupes $/ \mathrm{ks} /, / \mathrm{hm} /, / \mathrm{mm} /, / \mathrm{sk} / \mathrm{n}$ 'existent pas en malgache, et, pour pouvoir garder les phonèmes consonantiques français sans trop les altérer, le malgache dissocie généralement le groupe de consonnes en introduisant entre elles une voyelle épenthétique. D'où, par exemple, dans d'autres lexiques, ôkisizena «oxygène» au lieu de ôksizenina, girama «gramme» au lieu de grama ${ }^{3}$.

Certains auteurs de lexiques, comme le docteur Razafindrazaka il y a quelques années, ont fabriqué des mots nouveaux en tronquant certaines formes existantes. Ainsi, par la fusion phonique des éléments re «entendu, senti» et hetsika «mouvement», le lexicographe a créé retsika «nerf», réalisant de cette façon une combinaison phonologique nouvelle 4 .

La création lexicale résulte ici, au moins en partie, d'une combinaison phonologique inédite d'unités minimales, tout en portant aussi sur le rapport établi entre la matière phonique et la chose signifiée. 


\section{LA NÉOLOGIE MORPHOLOGIQUE}

La néologie morphologique est particulièrement productive dans la création terminologique en malgache, en raison de la propriété agglutinative que possède cette langue: elle consiste tantôt en la dérivation affixale, tantôt en la dérivation syntagmatique.

LA DÉRIVATION AFFIXALE

Dans un cadre morpho-lexical, on crée des lexèmes nouveaux par dérivation préfixale ou suffixale: 1) à l'aide des préfixes mp-, mpi-, mpan- ou mpana- indiquant l'agent habituel, par exemple, on a formé mpana-montsana (litt. «celui qui anéantit») «annulateur», mpan-ivo (de ivo «milieu, centre») «centralisateur», mpana-saraka (litt. «celui qui sépare») «discriminant», mpi-solo-tena (litt. «celui qui représente») 《représentant», dans le vocabulaire mathématique. Ce procédé de dérivation préfixale est utilisé pour créer une terminologie dans d'autres domaines. En électronique, on traduit «résonateur» par mpampiako (de mpampi- préfixe de l'agent causatif + ako «écho»). En statistique, on désigne «l'événement» par mpitranga (litt. «ce, celui qui habituellement se montre, se produit»); 2) le suffixe -ina ou -ana, morphème de conjugaison des formes verbales, n'a, en soi, que le rôle de simple accompagnateur du radical préfixé.

Très souvent, les processus de préfixation et de suffixation se cumulent dans un même vocable malgache.

Ainsi, «dérivation» (mathématique) se traduit par fisintonana (fi- préfixe de l'action verbale + sintona «action de tirer» + suffixe -ana). Or, les formes parasynthétiques sont fréquentes dans les langues austronésiennes, selon un phénomène que $\mathrm{E}$. M. Payne appelle la «circumfixation ${ }^{5} »$.

Il est intéressant de noter ici que les mots français porteurs des notions sont soumis à une analyse linguistique et à une interprétation sémantique avant que le créateur du terme malgache ne cherche empiriquement dans le lexique et dans la grammaire malgaches les éléments radicaux et affixaux susceptibles de les traduire, suivant les procédés du calque et du transfert sémantique ${ }^{6}$. C'est du moins là un des procédés morpho-lexicaux les plus classiques de création terminologique en malgache.

Sans doute, les procédés de dérivation affixale malgache varient d'une discipline à l'autre, et parfois d'un traducteur-terminologue à l'autre à l'intérieur d'une même discipline.

On observe ainsi, dans le lexique des mathématiques, une combinaison d'anciens morphèmes préfixaux à des radicaux déjà existants de la langue officielle et des dialectes, pour former des dérivés auxquels on n'aurait jamais pensé auparavant. Par exemple: fiatiana «inclusion», de aty «intérieur»; azo angambaina «probabilisable», de azo «que l'on peut» et de angamba «peut-être»; fimirana «égalité», de mira, terme dialectal signifiant «pareil, semblable ${ }^{\%}$.

En sciences physiques, pour décrire les phénomènes observés au cours des expériences, on emploie des néologismes munis des préfixes $/ \mathrm{m}$-/ des verbes agentifsstatifs et / $m p$ - / des substantifs déverbatifs d'agent habituel: «être en équilibre» se dit $m i$ toboka, de la forme dialectale toboka «état de ce qui est en repos, de ce qui est posé»; «solvant» mpandevona, litt. «celui qui dissout», de levona «consumé, anéanti, dissout, digéré».

Le transfert sémantique effectué sur ces néologismes devait fatalement aussi contribuer à enrichir le contenu sémantique du préfixal malgache.

Le vocabulaire médical malgache comporte également de nombreuses innovations morpho-lexicales originales: 1) le docteur Razafindrazaka a créé une nouvelle préfixation et une nouvelle suffixation hors des normes traditionnelles de la langue. Avec un morphème $k a$ exprimant généralement la défense dans certains dialectes, il adopte un préfixe $k a$ - exprimant l'antonymie: tsefotra litt. «petite explosion» /an-tsefotra «le 
sympathique» $/ k(a)$-an-tsefotra «le parasympathique». De même, il a cré un suffixe particulier, la lettre /-a/ (probablement un tronquement du suffixe/-ana/ ou /-ina/ de l'objectif) alternant avec un zéro, pour différencier des termes à radical commun: «métacarpe» tolokono / «métacarpien» tolokono-a. 2) Le docteur Edouard Randrianasolo, dans son ouvrage en 2 tomes $N y$ tenan' ny olombelona sy ny aretina (litt. «Le corps humain et les maladies») crée également de «nouveaux suffixes» malgaches: -sely «cyto» (cellule), -vihy «nucléo-» (noyau). C'est ainsi que dans la série lexicale de la cytologie, on trouve «cellule» sely/ «membrane cellulaire» hoditsely (hoditra «peau», + sely)/ «cytostome» vavatsely (vava «bouche», + sely), etc. Il s'agit là de nouveaux vocables à partir de calques sémantiques d'affixes savants français d'origine gréco-latine. Mais ces expressions, que l'on est tenté de ranger dans les cas de dérivation affixale, relèveraient plutôt en réalité de la dérivation syntagmatique, précisément de celle du syntagme d'apposition ${ }^{8}$.

LA DÉRIVATION SYNTAGMATIQUE

La complexité et la nouveauté des notions, pour le malgache, nécessitaient quelques formes d'approche des concepts, telles que la traduction en périphrases et la traduction en mots composés amalgamés, que Jacques Dez appelle, dans sa Syntaxe du malgache, le «syntagme d'apposition» et le «syntagme de dépendance».

LA DÉRIVATION SYNTAGMATIQUE EN PÉRIPHRASES

C'est là une des premières étapes linguistiques de la traduction de certains types de termes techniques français. La nouvelle terminologie malgache en périphrases consiste surtout souvent en une traduction-calque d'une définition ou d'une paraphrase du terme français.

Quelques syntagmes périphrastiques ont été fortement critiqués par des spécialistes et par des praticiens de chaque science et de chaque technique concernées, parce qu'ils ont été jugés trop longs, trop compliqués, parfois même sémantiquement incomplets et imprécis. Ce sont, par exemple: fiara manidina amin'ny bala afo manga (litt. «avion fonctionnant par feux d'artifices») pour traduire tout simplement «jet», et herim-baratra azo avy amin'ny toho-drano (litt. «énergie électrique obtenue à partir du barrage») pour rendre «énergie hydro-électrique».

Il fallait donc considérer seulement la traduction en syntagmes périphrastiques comme une première étape de la lexicalisation. Cependant, toutes les terminologies malgaches actuelles comportent encore des périphrases.

LES SYNTAGMES D'APPOSITION ET LES SYNTAGMES DE DÉPENDANCE

Ces expressions, qui appartiennent à la terminologie linguistique de la Syntaxe $d u$ malgache, sont les seules adéquates pour dénommer avec précision la façon dont les Malgaches désignent les concepts techniques par des formations composées amalgamées (kambanteny, litt. «mots associés»). Il s'agit de nouveaux syntagmes constitués d'éléments libres placés côte à côte et qui se comportent syntaxiquement comme des signifiants simples.

Le vocabulaire mathématique et une grande partie du vocabulaire d'autres disciplines comportent de nombreux syntagmes d'apposition, du type savazoro «diagonale» (sava «action de séparer en deux» et zoro «angle»), ou du type haja mampitovy «isomorphisme» (haja «respect» et mampitovy «qui compare, qui rend égal ${ }^{9} \gg$ ). Ce ne sont pas toujours des calques sémantiques du français, mais des associations intuitives de termes anciens, qui, intégrées par transfert sémantique dans un champ lexical nouveau, évoquent la notion désignée.

Les syntagmes de dépendance, marquant la dépendance d'un objet par rapport à un autre, se rencontrent surtout dans les formations composées contenant des noms propres: «théorème de Pythagore» raikitr'i Pythagore (raikitra, litt. «ce qui a été décidé, conclu à 
titre de prémisses»), «détroit de Haller» andilan'i Haller. Mais on en trouve aussi dans les expressions de phénomènes physiques qui sont en relation de dépendance les uns par rapport aux autres : ainsi, «pression atmosphérique» se dit tsindrin'ny atimosifera, dont le deuxième terme à valeur génitive, atimosifera, est relié à la base tsindry "pression" par le morphème fonctionnel de la possession $n(a)$. Là encore, le transfert sémantique intègre les termes dans le champ nouveau des significations spécialisées.

Toutes ces formations néologiques à caractère grammatical impliquent, en fait, une interférence évidente du grammatical et du lexical: par le jeu du système phonologique et de la morphologie, on crée des unités lexicales nouvelles dont le sens est le résultat d'un transfert sémantique.

\section{LES FORMATIONS NÉOLOGIQUES À CARACTERE LEXICAL}

En traduisant du français technique et scientifique en malgache, on choisit souvent dans cette dernière langue des termes du vocabulaire général, et on en fait des néologismes sémantiques par transferts de signification: transferts métonymiques ou transferts métaphoriques. Et ce sont les définitions des nouveaux termes obtenus qui fixent cette fois-ci leurs signifiés.

Parmi les autres formations néologiques à caractère lexical, nous citerons également les emprunts aux langues européennes, aux dialectes malgaches, et la traductioncalque.

LES TRANSFERTS MÉTONYMIQUES

Il s'agira de faire de certains termes anciens des termes nouveaux, en restreignant ou en étendant leurs sens selon les besoins du traducteur-terminologue.

C'est dans le vocabulaire des mathématiques et des sciences de la nature qu'on observe surtout le phénomène de métonymie ou de synecdoque. L'exemple du terme dialectal lio, litt. «sang» est très significatif de ce point de vue: par transfert de signification, il sert à désigner maintenant les «globules du sang». C'est ainsi que nous avons: lio mena «globules rouges» et lio fotsy «globules blancs». De même, par généralisation (ou par extension) du sens des termes anciens, on fait du terme dialectal teza, litt. «cœur du tronc d'arbre», l'équivalent malgache d'«axe» (mathématique).

LES TRANSFERTS MÉTAPHORIQUES

Les transferts métaphoriques s'effectuent également par spécialisation du terme, par changement et extension du sens. Tsilo, litt. «épine, flèche d'indien» devient alors la traduction mathématique de «vecteur», et mampita, qui avait le sens concret et littéral de «faire passer l'eau, faire franchir, transporter au-delà» rend le sens mathématique de «transitif».

Il n'est évidemment pas toujours possible de traduire une métaphore française par une nouvelle métaphore malgache, en raison des différences culturelles qu'elles comportent, et on s'en tient souvent au seul sens technique. Le «tendon d'Achille» (terme de connotation mythologique) est traduit par le révérend P. Malzac (Dictionnaire, 1888) traingovazana «le tendon du jarret» et par le docteur Razafindrazaka kirohitraingo, litt. «lien-muscle au-dessus du talon».

LES DÉFINITIONS

Ce sont alors les définitions des termes, qui, finalement, déterminent les signifiés. Elles peuvent être identifiées soit dans une grande partie de la forme linguistique des néologismes (ex. : «matrice symétrique» vohoka mira amin' ny tsimbadiny, litt. «matrice égale (à elle-même) dans l'état où elle se trouve lorsqu'elle est retournée, inversée ${ }^{11}$ », soit dans quelques éléments lexicaux qui évoquent la notion (ex. : «nerf» tatitra, litt. «message», «nerf moteur» tatibaiko «message transmis sous forme d'ordres venant du cerveau», «nerf sensitif» tatibolana «message transmis du corps au cerveau»). 
La valeur d'une traduction d'une terminologie française en malgache dépend, en tout cas, de la solidité des définitions attribuées par les terminologues aux néologismes. - Les autres formations néologiques: les emprunts

Il semble évident que les emprunts ne sont souvent que des palliatifs à des carences lexicales. Or, par sa tradition historique, la langue scientifique et technique malgache ne pouvait emprunter certains termes savants qu'au français, et, dans une moindre mesure, à l'anglais. Toutefois, étant donné la découverte de la richesse des dialectes malgaches, les emprunts dialectaux se révéleront aussi de plus en plus nombreux.

- La néologie par emprunt aux langues européennes

Des emprunts au français, généralement des noms, des substantifs et des adjectifs, ont été adoptés dans les lexiques spécialisés malgaches. Ils présentent d'ailleurs un certain nombre de caractéristiques au point de vue phonétique, morpho-syntaxique et sémantique.

Au point de vue phonétique, ces emprunts ont subi une malgachisation plus ou moins complète, selon les cas.

Quelques-uns, des mots courts et leurs dérivés, ont été intégrés totalement. Par exemple, la transformation de «mètre» en metatra, de «litre» en litatra, de «centimètre» en santimetatra, a été facilitée par le fait que le groupe final «consonne $+\mathrm{r}$ » du français tel que $/ \mathrm{br} /, / \mathrm{vr} /, / \mathrm{tr} /$ est perçu en malgache comme l'équivalent de «consonne + voyelle -tra» du malgache. D'autres termes, plus longs, ont été adoptés, moyennant des dérogations aux incompatibilités phonologiques du système malgache, telle la rencontre de 2 consonnes (cf. supra «La néologie phonologique») (ex. : prôtida «protide», psikôlồjia «psychologie», vaksiny «vaccin», etc.).

D'autres n'ont pas du tout été malgachisés et gardent leur orthographe originelle en entrant dans le lexique malgache. Au point de vue morphologique, les suffixes de certains adjectifs français en «-ien» et en «-ique» ont été retranscrits phonétiquement en -iana et en -ika: «algébrique» alijebrika, «abélien» abeliana, «cartésien» kariteziana, ce qui semble enrichir le système suffixal déjà existant dans la langue.

Au point de vue sémantique, une des caractéristiques de l'emprunt dans la traduction scientifique et technique en malgache est de garder le caractère monosémique et monoréférentiel du signe français. Les faux amis sont rares, voire inexistants. On observe alors des ressemblances formelles et une identité de contenu entre les mots français et les emprunts malgachisés.

L'emprunt malgachisé est donc bien, en fin de compte, un composé de deux conventions linguistiques différentes, selon un dosage variable.

La plupart des mots empruntés sont familiers pour les utilisateurs malgaches, généralement bilingues. Mais ils sont relativement peu nombreux et ils se distinguent d'ailleurs de l'ensemble des termes du lexique commun du malgache par certains caractères orthographiques et morphologiques particuliers.

LES EMPRUNTS DIALECTAUX

La traduction scientifique et technique du français en malgache a fait largement appel aux emprunts dialectaux, depuis que l'on s'est rendu compte des potentialités des dialectes malgaches.

Les régions côtières, par exemple, ont fourni des vocables pour la terminologie de la mer, de la pêche et de la navigation (ex. : hoala «golfe», lovoka «petite baie», helodrano «baie», banja «bassin», deboka «dépression, cuvette», dengy «fosse», etc.). Les Hauts-Plateaux connaissent les nuances du vocabulaire de l'orographie (ex. : bongo «buttes, dômes», vozontany «col», havoana "colline», zohy «grotte», lemaka "plaine», harambato «rocher», etc.12). Le sud de Madagascar, à vocation pastorale, la côte orientale 
et septentrionale, réputés pour leur faune et leur flore, sont aptes à enrichir la terminologie malgache de la zoologie, de la zootechnie, de la botanique, des eaux et forêts.

Un certain nombre de vocables malgaches d'origine dialectale est entré dans la langue des mathématiques (mira «égal», teza «axe», teboka «point», etc.), de la physique (zaka «matière», haabo «niveau», haambo «altitude», miiba «négatif», miabo «positif»), de la biologie (marovihy «polynucléaire», lio «globules»), de la linguistique (vara «prédicat», etc.).

Le docteur Razafindrazaka, pour sa part, a beaucoup emprunté au parler antandroy de Tolagnaro (Fort-Dauphin) pour élaborer son vocabulaire médical : en effet, l'antandroy possède un lexique très varié relatif à l'organisme et au corps humain. C'est de là qu'il a, par exemple, tiré les termes sitry «sacrum», ohy «coccyx», dalia «rectum», kono «carpe», zaty «tarse», kapemba «thymus», etc., et une partie du système préfixal dont il avait besoin pour différencier les concepts 13 .

Tout un travail est encore à l'étude pour exploiter davantage et plus systématiquement le vocabulaire des dialectes malgaches.

LES TRADUCTIONS-CALQUES

Les traducteurs-terminologues ont fait largement usage d'une autre forme d'emprunt: le calque sémantique. Ils y voient l'avantage d'enrichir et de moderniser la langue malgache par l'introduction de concepts nouveaux, et de reconstruire le sens des vocables français tout en se servant du lexique malgache.

Une grande partie des différentes terminologies techniques malgaches comportent des traductions-calques. C'est, en effet, une façon, non plus de faire des emprunts formels, mais de faire appel aux possibilités de création offertes par le système lexical de la langue malgache pour substituer un terme national au terme européen tout en désignant le même signifié. Ainsi, l'IREM a utilisé vohoka, qui signifiait primitivement «matrice, sein, utérus» pour traduire «matrice», au sens mathématique. Le calque permet, de cette façon, de maintenir dans une forme nationale un lien conceptuel avec les terminologies étrangères à valeur internationale.

Cependant, l'abus du calque sémantique pose des problèmes gênants, lorsque les dénominations françaises et malgaches impliquent, les unes par rapport aux autres, des différences de connotations et des possibilités d'écart sémantique ou socio-linguistique. Le professeur Raoelina-Andriambololona l'a signalé dans un article, à propos des confusions dans la traduction malgache de la notion mathématique de «fonctión ${ }^{14} »$.

C'est pour cela que différentes commissions de traduction ont souvent dû procéder autrement que par le calque, notamment la Commission de la langue juridique malgache et celle des sciences physiques.

Dans le domaine de la langue du droit, on a même sensiblement dépassé le stade de la simple traduction-calque des termes. En effet, la langue juridique a un caractère plus ou moins «littéraire», et demande un choix approprié des termes pour bien traduire les intentions et la pensée du législateur. La Commission de la langue juridique malgache a donc décidé de se consacrer, dans un premier temps, à la traduction intégrale d'un certain nombre de textes fondamentaux, puis, dans un deuxième temps, au classement, dans un Rakibolana (dictionnaire malgache), de la terminologie adoptée.

La terminologie juridique des Rakibolana, en ce sens, semble exiger avant tout une explication des termes français dans leur contexte et un éclaircissement sur les situations de droit qu'ils désignent. Par exemple, le terme polysémique «droit» a été traduit différemment selon les circonstances de son emploi : 1) rariny, pour la justice sociale, 2) zo, pour les droits civiques, 3) lalana, pour la loi, 4) fahefana, pour les pleins pouvoirs ou pour le droit d'agir, 5) fahazoana pour l'autorisation de faire quelque chose. Ce sont là 
des termes usuels, mais qui, dans la terminologie juridique, tiennent compte de la situation d'emploi ou des environnements linguistiques des termes utilisés.

On sait que la langue juridique malgache possède une assez longue tradition qui remonte au XIXe siècle, à l'époque du Code des 305 articles. En dehors des emprunts au français (ex. : kadasitra «cadastre») et de quelques traductions-calques (ex. : lalanalasitra «loi-cadre», de lalana «loi», et de lasitra «moule, cadre»), ce sont des vocables simples du vieux malgache, tombés en désuétude (ex. : sara «péage, frais»), ou du malgache quotidien (onitra «réparations d'un tort» repris avec le sens de «dommagesintérêts»), qui ont été le plus souvent réemployés avec un sens moderne pour rendre la signification précise des termes juridiques donnés par le français.

Le vocabulaire juridique malgache ne recourt plus très souvent à la traductioncalque, et il a l'avantage de pouvoir renouveler le contenu des signifiants déjà existants et de lui donner une précision technique adaptée aux circonstances et aux textes de lois actuels.

Il serait intéressant aussi de voir comment les terminologues malgaches des sciences physiques procèdent en évitant la facilité et les inconvénients du calque sémantique.

D'après nos enquêtes, en 1978, sur les méthodes de travail des équipes spécialisées en physique ${ }^{15}$, la démarche intellectuelle des auteurs de manuel et des terminologues suit les étapes suivantes: 1) description des réalités et des phénomènes physiques observés; 2) élaboration des concepts et des définitions; 3 ) recherche des dénominations malgaches jugées appropriées à ces concepts et à ces définitions; 4) «contrôle» par les expressions françaises correspondantes et élaboration d'un glossaire (vakobolana).

Soit, par exemple, la notion de «niveau». Après observation, le terminologue perçoit le concept à partir d'une réalité qu'il définit comme «la limite de la surface supérieure de l'eau» (faritry ny velatra anabon'ny ranoka); puis il donne à la notion la dénomination de haabo (terme d'origine dialectale) dont il contrôle le sens par l'équivalent français «niveau».

En d'autres termes, dans les premiers temps de la malgachisation, la démarche semblait imposer, au départ, une étude des phénomènes physiques, puis celle des concepts, avant d'aborder les problèmes linguistiques des définitions, des dénominations et du «contrôle» par la traduction. On peut penser d'ailleurs que la définition formulée en malgache se confond avec l'analyse de l'objet auquel se réfère la nouvelle dénomination malgache.

Cette série d'opérations mentales, qui fait table rase de beaucoup de notions reçues et des habitudes de la traduction-calque, ne fait appel au contrôle de la traduction - si traduction il y a - qu'a posteriori, et c'est ce qui fait l'originalité de la recherche terminologique en sciences physiques.

CONCLUSION

Nous avons essayé de rendre compte ici des tendances et des grandes lignes toujours actuelles - de la création terminologique en malgache, ainsi que des tentatives de solutions. Il reste à savoir maintenant comment cette terminologie circule et est diffusée auprès du public.

\section{PROBLÈMES DE DIFFUSION DES TERMINOLOGIES SCIENTIFIQUES \\ ET TECHNIQUES MALGACHES}

Bien entendu, il ne suffit pas pour les traducteurs-terminologues d'apporter des innovations à la langue ni de créer des néologismes savants. Il faut aussi que ces terminologies soient diffusées pour voir si elles sont utilisées d'une façon effective et durable. Or la question est de savoir qui sont les utilisateurs réels ou potentiels de ces terminologies, et quel usage il en a été fait. C'est là que nous examinerons tour à tour les 
conditions de diffusion et d'utilisation des néologismes scientifiques dans le passé, les problèmes actuels de diffusion et l'état présent du travail terminologique.

\section{HISTORIQUE DE LA DIFFUSION DES TERMINOLOGIES}

Il semble nécessaire ici de faire un rappel historique des conditions de diffusion des créations terminologiques à Madagascar, pour donner une certaine idée des problèmes que pose la vulgarisation des néologismes technico-scientifiques.

Ainsi, dès le XIX ${ }^{\mathrm{e}}$ siècle, le travail des missionnaires de Londres était déjà une des premières tentatives de malgachisation et de diffusion de la langue scientifique: 1'utilisation du malgache comme langue d'enseignement était complétée, à l'époque, par la publication, dans cette langue, de manuels de botanique, de géologie et de mathématiques ${ }^{16}$. Mais, avant 1895 , étant donné le nombre relativement restreint de gens lettrés et instruits, on suppose que les manuels scientifiques et techniques d'enseignement supérieur en malgache à l'usage de 40 élèves de Faravohitra et des étudiants en médecine d'Ankadinandriana ${ }^{17}$ ne pouvaient encore diffuser leurs terminologies que dans ce cercle limité de la jeunesse intellectuelle malgache. Et toutes ces entreprises, si nombreuses fussent-elles, avaient dû être interrompues et même complètement oubliées pendant plus d'un demi-siècle.

C'est en 1964 que le premier Rakibolana, rassemblant les expressions juridiques malgaches tirées des traductions, fut édité par une commission de la langue juridique et technique malgache du ministère de la Justice. Il devait être complété, en 1976, par le Vocabulaire français-malgache, Mots juridiques et techniques avec exemples d'Armand Rakotovao, magistrat et membre de l'Académie malgache. On constate alors que, dans le domaine juridique, les termes du Rakibolana sont entrés dans la pratique sociale, comme en témoignent les actes des tribunaux, les textes du Journal Officiel, les notes et circulaires des ministères en langue malgache. Le Rakibolana français-malgache d'Armand Rakotovao (EESDEGS 1977) illustre d'ailleurs la façon la plus claire et la plus accessible d'utiliser en malgache la terminologie juridique (dont les signifiants sont ceux de la langue usuelle).

En 1965, une commission du ministère de l'Agriculture publia dans les Mémoires de l'Académie malgache une traduction en langue malgache des termes techniques, qui devait répondre au besoin pratique de vulgarisation des techniques agricoles en milieu paysan. L'idée était d'établir une communication simple entre les experts, les techniciens qui servent d'intermédiaires et les paysans, avec un vocabulaire issu de la langue usuelle du milieu. Or, la malgachisation de la langue technique de l'agriculture, de l'élevage, du paysannat, des eaux et forêts, était alors encore à l'état d'expérimentation. Il semble normal, par exemple, que la réalisation d'un «compost» (traduit zezipako, litt. «engrais à partir de détritus»), avec la participation des paysans, demandât des explications techniques en termes ordinaires sur l'utilisation et le dosage des matériaux organiques et des formes d'énergie existantes.

Depuis 1973, la terminologie malgache des mathématiques, des sciences physiques et biologiques, est diffusée dans les manuels en usage dans les écoles et pratiquée déjà par quelques générations de jeunes gens.

Parmi les publications, nous citons: Kajy («calcul»), Marika vaovao («mathématiques modernes»), Voambolana (lexique des mathématiques, IREM, ministère de l'Éducation nationale et des Affaires culturelles), Lefan' ova tokana (litt. «fonction unique à variables», de Pierre Rajaona, IREM, Université de Madagascar, 185 p.), Voambolana ifotoran'ny fampianarana tekinika iraisana ao amin'ny kilasy valohany sy faharoa («lexique de l'enseignement technique $1^{\text {re }}$ et $2^{\mathrm{e}}$ années», concernant l'habillement, les constructions métalliques, le bâtiment, la menuiserie, 1977), Fizika («physique», classe 
de 6e, de Martial Andrianaivo, Éditions Papigile de la Grande Île, 1976), Fizika-simia (classe de 5e, ministère de l'Éducation nationale, 1977, 132 p.), Fizika-simia $\left(5^{\mathrm{e}}\right.$ et $4^{\mathrm{e}}$, de Martial Andrianaivo, Éditions Salohy, 1976), Fizika (classe de 6e, de P. Raminosoa, Fofipa, 1976), Fahalalana Tsotra («leçon de choses», classes de $7 \mathrm{e}$ et $8 \mathrm{e}$, d'André Rakotondranaivo, Éditions Papigile, Tananarive, 1975, 123 p.), Ny tenan' ny olombelona sy ny aretina ( «le corps humain et les maladies», d'Edouard Randrianasolo, tome I-1975, tome II-1976, tome III et IV-1978).

En février 1987, 1'imprimerie du CNAPMAD a fait un tirage à 20000 exemplaires d'un «lexique malagasy-malagasy-français» (Service de logistique pédagogique) regroupant la terminologie par ordre alphabétique de 42 disciplines différentes: agriculture, bâtiments-travaux publics, chimie, cuisine, dessin mécanique, dessin technique, école des arts et techniques, économie, électronique, électrotechnique, éducation physique et sportive, fabrication mécanique, secrétariat, géographie, gestion, géologie, habillement, histoire, hydraulique, imprimerie, installations sanitaire et thermique, linguistique, mécanique automobile, métaux en feuilles, mathématiques, mécanique, métallurgie, météorologie, musique, ouvrage bois, ouvrage métallique, pédagogie, philosophie, physique, puériculture, psychologie, résistance des matériaux, sciences naturelles, statistique, technique, topographie, traitement thermique. C'est, en somme, une compilation et une mise à jour en 235 pages des résultats de tous les travaux terminologiques effectués jusqu'en 1987.

Cependant, devant ce foisonnement de terminologies nouvelles, durant ces dernières années de malgachisation, des problèmes de choix, d'assimilation, d'acceptation ou de refus des néologismes malgaches se posaient. Déjà aussi se dessinaient chez les enseignants utilisateurs et chez les spécialistes, deux tendances opposées: 1) les partisans d'une malgachisation des emprunts européens (au français, par exemple), dans le souci de ne pas troubler la vocation internationale des nouveaux lexiques malgaches; 2) les partisans d'une malgachisation totale, autant que faire se peut, de la terminologie scientifique et technique, afin de rendre à la langue sa pureté initiale.

$\mathrm{La}$ première tendance était représentée par le professeur RaoelinaAndriambololona, dont les articles, parus dans le Bulletin de l'Académie Malgache en $1976^{18}$, montraient une position ferme contre la malgachisation à outrance.

La deuxième tendance, représentée par certains artisans de la malgachisation (dont le docteur E. Randrianasolo, par exemple), était motivée par un besoin urgent de mots malgaches pour désigner les notions venant d'Occident (notions mathématiques, techniques, notions de physique, de biologie, etc.). Et il arrive même que ce besoin soit si fort, dans le contexte de la malgachisation qui touche certains domaines (enseignement, administration, échanges dans la vie quotidienne, etc.) que certains milieux malgaches accepteraient peut-être des mots mal faits, pourvu que ces mots soient malgaches. D'où l'affirmation, qui a sans doute besoin d'être nuancée, du docteur Randrianasolo:

Les termes scientifiques et techniques malgaches, même les plus mal faits, sont préférés par les Malgaches aux meilleurs termes scientifiques et techniques étrangers ${ }^{19}$.

Devant diverses tendances dans la conception même de la terminologie malgache, et en l'absence de normalisation académique, la question embarrassante demeurait alors celle de savoir quelle terminologie adopter dans la langue d'enseignement ${ }^{20}$.

\section{LES PROBLÈMES ACTUELS DE DIFFUSION}

Il se pose alors actuellement des problèmes difficiles de diffusion des terminologies: non seulement les réactions du public devant les innovations lexicales sont diverses, mais encore les nécessités d'une normalisation s'imposent. 
LES RÉACTIONS DU PUBLIC

La diffusion des néologismes techniques et scientifiques soulève des réactions diverses du public. D'une part, on a examiné les problèmes de vulgarisation des néologismes juridiques et économiques malgaches, d'autre part, ceux des termes techniques des manuels scolaires de mathématiques, de physique, de sciences naturelles et de grammaire.

Un fait est certain: la langue juridique malgache des Rakibolana a été conçue à l'usage du grand public, précisément parce que les expressions ressenties comme techniques et savantes dans la langue française ont été le plus souvent traduites en malgache par des expressions usuelles.

Ainsi, certaines formules juridiques archaïques et stéréotypées en français, telles que «réserve les dépens», ont été rendues en malgache par des équivalents apparemment plus explicites et plus accessibles aux usagers: tsy mbola lazaina aloha izay vola lany amin' ny fitsarana «les frais de tribunal ne sont pas encore connus d'avance».

En réalité, le problème n'est pas aussi simple qu'il le paraît, parce qu'il se situe moins au niveau de la transmission des signifiants usuels qu'au niveau de la compréhension totale des concepts juridico-économiques par le grand public. En effet, les termes juridiques malgaches que l'on croit bien comprendre restent des néologismes sémantiques: on met à égalité une expression malgache et une expression juridique française alors que cette égalité n'existait pas auparavant. Et le phénomène de l'effet Thémis se manifeste nécessairement.

Par exemple, il n'est pas certain que l'expression teny midina litt. «paroles qui descendent (d'en haut)» soit comprise immédiatement et entièrement par tous dans le sens précis de «circulaire» émanant d'un supérieur hiérarchique et adressée au personnel sous ses ordres. Et la vulgarisation des traductions malgaches des conventions diplomatiques et économiques au Journal Officiel soulève le même problème: l'emploi des termes très généraux pour traduire des notions très précises ne facilite pas toujours la compréhension des lecteurs, en raison des pièges de la polysémie qu'il introduit. Ainsi, peut-on toujours comprendre pleinement l'expression fitotonganan'ny vidin'ny akorambokatra («chute du prix des récoltes») dans le sens voulu par le traducteur de «détérioration des termes de l'échange»?

C'est donc là que s'impose toute une initiation juridique et économique en malgache qu'impliquent tous les transferts sémantiques observés dans la terminologie.

La diffusion des néologismes scientifiques et techniques des manuels scolaires de mathématiques, de physique, de sciences naturelles et de grammaire soulève également des problèmes sérieux. Ceux-ci se situent au niveau du public visé et de l'efficacité pratique.

Les terminologies des disciplines précitées ont circulé dans les écoles et elles ont été utilisées par quelques générations de jeunes gens entre 1978 et 1990. Mais déjà quelques observateurs sévères et hâtif y voient surtout une circulation et une communication en vase clos: de l'enseignant aux élèves qui renvoient à l'enseignant l'image du langage «ésotérique» de son enseignement. Et semble être exclue du dialogue la masse de parents d'élèves, qui n'arrivent plus à suivre leurs enfants dans leurs études de grammaire ou de mathématiques, parce qu'ils ne disposent même pas tous des lexiques spécialisés qui, d'ailleurs, sont en nombre insuffisant actuellement faute d'une plus large diffusion.

LA NORMALISATION DES TERMINOLOGIES

Il n'existe pas encore de normalisation académique de la terminologie, bien que l'Académie malgache ait depuis longtemps réuni des commissions spécialisées en diverses terminologies et en divers domaines et les ait présidées (langue juridique, 
technique, langue de l'agriculture et des eaux et forêts, etc.), et qu'elle soit encore en train de réaliser un projet de dictionnaire.

Or, nous avons évoqué plus haut les problèmes de dispersion des terminologies ${ }^{21}$ en raison de la diversité des publications de terminologues travaillant parfois isolément et d'une façon empirique et hâtive, sans s'être informés du travail terminologique qui s'est fait dans le monde entier depuis quelques années. D'aucuns parlent même d'un «bricolage» terminologique dans les premiers temps de la malgachisation, en doutant du succès d'une telle entreprise 22 au niveau de la diffusion de ce travail et de sa normalisation nationale. Les tâtonnements ont été nombreux.

Il n'y a pas encore eu un accord officiel entre producteurs et usagers pour unifier la terminologie, mais on peut peut-être déjà considérer comme un début de normalisation de fait l'usage répandu des terminologies des travaux qui semblent faire autorité jusqu'à présent (ceux de l'IREM, du Service de logistique pédagogique et du Fahalalana Tsotra d'A. Rakotondranaivo).

Après l'expérimentation des terminologies de ces dernières années, le moment est venu, depuis 1988, de faire le bilan du travail terminologique réalisé à Madagascar, afin de rechercher une nouvelle stratégie de développement des activités terminologiques. C'est le but que s'est proposé le Centre de recherche sur les traditions orales et les langues nationales africaines en Afrique orientale (EACROTANAL), en collaboration avec l'Académie malgache, à l'occasion d'un séminaire d'information sur les aspects méthodologiques et pratiques du travail terminologique (Antananarivo, 12-21 décembre 1988).

\section{ÉTAT PRÉSENT DU TRAVAIL TERMINOLOGIQUE}

Après ces années de tâtonnement tant au point de vue de la création lexicale que de la diffusion des ouvrages scientifiques et techniques dans la langue nationale, le travail terminologique malgache en est maintenant à l'heure des bilans. Il s'agit de tirer des leçons de ces aventures et de ces expériences terminologiques des dernières années, et de voir, dans le contexte des expériences faites par-ci par-là dans d'autres pays francophones ou non francophones, comment unifier et harmoniser les terminologies.

UN TRAVAIL TERMINOLOGIQUE DANS L'ESPACE FRANCOPHONE

Il n'est pas question ici de faire un panorama complet de l'activité terminologique de langue française sur le plan international, mais de recenser très rapidement certains travaux et projets de terminologie en $1988^{23}$ dont les préoccupations se rapprochent de celles des terminologues malgaches. Nous citons, par exemple:

- Dans le domaine du droit et de la législation: Dictionnaire juridique anglais-français. Responsable: Jack A. Clarence Smith (17 000 notions) (Québec, ministère de la Justice, Bureau des règlements).

- Dans le domaine de l'agriculture et du génie génétique: Arrêté de terminologie (agriculture). Responsable: Commission ministérielle de terminologie de l'agriculture. Environ 100 notions. Vocabulaire français-anglais. Travail sur support informatique (France, ministère de l'Agriculture)

- Dans le domaine de la cartographie, de la photographie aérienne: Cartographie et photographie aérienne. Travail rédigé par A. Supply. Vocabulaire espagnol-français (Mémoire de licence en traduction. Centre de terminologie de Bruxelles).

- Dans le domaine de la linguistique: Vocabulaire de la linguistique. Responsable: Yves Gilly (Université de Franche-Comtê, UFR des sciences du language, de l'homme et de la société). 
Nous notons un ouvrage déjà publié en 1983, et réédité par l'AUPELF et EDICEF: «Inventaire des particularités lexicales du français en Afrique noire» par l'équipe IFA. L'intérêt de cet ouvrage réside dans la réhabilitation et dans la valorisation d'un vocabulaire inspiré du français mais qui a acquis une spécificité en raison de ses écarts par rapport à l'usage du français standard.

Tous ces ouvrages de référence peuvent déjà constituer, de près ou de loin, une des bases de réflexions pour les terminologues malgaches.

UN TRAVAIL TERMINOLOGIQUE EN AFRIQUE. L'EXEMPLE ÉTHIOPIEN

Une confrontation des expériences faites dans quelques pays d'Afrique orientale et de l'océan Indien (Tanzanie, Éthiopie, Comores, Maurice, Madagascar) s'est révélée aussi stimulante et éclairante, au cours du séminaire d'information de l'EACROTANAL. L'exemple éthiopien a servi de base de comparaison des expériences ${ }^{24}$.

En Éthiopie, les terminologies reposent essentiellement sur la traduction des termes scientifiques et technologiques anglais en amharique. L'objectif est de transmettre les connaissances étrangères et de permettre aux masses de comprendre les termes étrangers au moyen de traductions dans la langue autochtone. Ainsi, le travail terminologique éthiopien s'insère dans un vaste projet de vulgarisation de masse en matière de science et de technologie.

En ce sens, le gouvernement éthiopien a reçu, dès 1981, une aide du fonds des Nations unies pour la science et la technologie au service du développement (UNIFSTD) : le projet a été intitulé «Développement de la capacité nationale à la vulgarisation et à la formation en science et en technologie au moyen de la langue et de la démonstration».

À peu près à la même époque, l'Académie éthiopienne s'est vu confier la responsabilité d'exécuter le Projet de traduction de termes scientifiques et technologiques en amharique (STTTP). Les travaux ont été répartis entre 13 comités (agriculture, botanique, bâtiment et construction, chimie, électro-mécanique, géographie, géologie, mathématiques, médecine, diététique, physique, statistique, zoologie), comprenant chacun un linguiste et deux spécialistes de la discipline. Les activités des comités ont été coordonnées par un directeur, un expert linguiste en chef et deux coordonnateurs techniques. Cela implique des objectifs à long terme et immédiats.

À long terme, l'objectif principal a été de fournir une terminologie standardisée par l'intermédiaire de laquelle la connaissance scientifique et technologique pourra être facilement diffusée et assimilée à tous les niveaux de la société éthiopienne.

Parmi les objectifs immédiats, il a fallu traduire les termes techniques d'autres langues en amharique, suivant les procédés de l'emprunt (au grec, au portugais, au français, à l'italien et à l'anglais), de la création néologique, de la combinaison de mots, de l'extension de sens et de l'adaptation.

Puis il a fallu préparer des ouvrages populaires qui utilisent des termes techniques amharique les plus simples, des dictionnaires scientifiques, des fascicules illustrés, disponibles pour une consultation des 80 participants au séminaire international de terminologie ${ }^{25}$. Et l'Académie des langues éthiopiennes se charge de son propre programme de vulgarisation et de révision des terminologies: diffuser les termes auprès des principaux usagers et, au moyen des échos obtenus, normaliser les nouveaux termes et s'assurer qu'ils sont effectivement utilisés selon les besoins.

Il est intéressant de noter que dans une partie de l'espace francophone comme en Afrique non francophone, la base de beaucoup de recherches en terminologie est la traduction. Mais le problème des pays développés semble être de rechercher la façon de normaliser les termes traduits ${ }^{26}$, alors que celui de certains pays africains se concentre beaucoup plus encore sur la traduction des termes de base et sur leur vulgarisation. 
BILAN DU TRAVAIL TERMINOLOGIQUE MALGACHE

Une série de leçons méthodologiques, diffusées à partir des expériences de plusieurs pays en matière de terminologie, permet aux lexicographes malgaches de situer leurs travaux par rapport à ceux du monde francophone et non francophone. Mieux encore: l'échange d'expériences au cours du séminaire d'information de l'EACROTANAL a rassuré les terminologues malgaches qui se sont rendu compte qu'ils n'avaient pas fait fausse route. Les participants étrangers de ce séminaire, d'après leur évaluation, ont même reconnu que Madagascar compte parmi les pays africains avancés en ce qui concerne la promotion et l'utilisation des langues nationales dans le domaine de l'éducation et de l'enseignement scientifique et technique. Seulement, les participants nationaux ont aussi pris conscience d'un manque de planification, de coordination dans leurs travaux et de réseaux organisés en matière d'échanges d'information et de système de diffusion des résultats de ces travaux.

A) Quels sont les travaux terminologiques qui ont été diffusés jusqu'à présent et qui ont été retenus au cours de ce séminaire? Ce sont généralement des lexiques publiés ou diffusés à travers l'enseignement :

- Le vocabulaire technique des eaux et forêts, qui fait suite à «la traduction en langue malgache des termes techniques par la Commission du ministère de l'Agriculture» (Mémoire de l'Académie malgache, vol. XLI, 1965).

- Le lexique des mots et expressions du Journal Officiel. On y remarque encore la diversité des traductions, en raison des différences dans la formation des traducteurs (administrateurs civils, juristes, économistes, financiers, douaniers, enseignants littéraires, etc.).

- La terminologie de la grammaire transformationnelle adaptée au malgache. Aucun lexique n'a été publié encore, mais un certain nombre de termes ont été traduits et circulent depuis peu dans le milieu des étudiants malgachisants. Ce sont, par exemple : «transformation» = fiofohana (de miofo «changer de couleur»), «génération» = fianahana (litt. «enfantement», de anaka «enfant»), «intuition»= saina tselatra (litt. «intelligence éclair»), «syntagme» = tarika (litt. «groupe»), «prédicat» = vara (terme dialectal signifiant «ce que l'on dit»), etc.

Cette terminologie est couramment pratiquée par certains candidats au mémoire de maîtrise et de CAPEN en malgache, dirigés par Roger-Bruno Rabenilaina à l'École normale supérieure d'Antananarivo. L'enseignement sert ici de terrain d'essai et de moyen de diffusion.

- Le lexique malgache de la cartographie, de la géodésie, de la géographie, de la photogrammétrie et des photographies aériennes, de la topographie.

Les termes malgaches de la cartographie circulent déjà dans les brochures touristiques bilingues décrivant la configuration géographique des régions de Madagascar. Ainsi, un certain nombre de termes de la cartographie sont connus du public, tels que: «échelle» = maridrefy (de marika «plan, dessin» et refy «mesure»); «projection» = fanipazana (de manipy «lancer, jeter»); «croquis» = kisarisary (litt. «petits dessins»), etc.

- Le lexique des mathématiques, appliqué depuis 1973 dans l'enseignement des mathématiques en malgache.

- La terminologie technique appliquée dans l'enseignement technique en malgache.

- Les terminologies scientifiques dans le domaine de l'avifaune à Madagascar.

C'était l'objet d'une thèse de doctorat de Marguerite Razarihelisoa. Dans un compte rendu paru dans les publications du Centre d'études et de recherches sur l'océan Indien n $^{\circ} 3$ (Paris, INALCO, 1988, p. 553), l'auteur mentionne avoir donné une liste systématique des oiseaux de Madagascar en recueillant les termes des nomenclatures en latin, en français et en anglais, ainsi que les noms malgaches de la langue officielle et des 
dialectes. Elle procède à un certain nombre de remarques linguistiques (sémantiques, morphologiques, phonologiques) sur les noms malgaches des oiseaux selon quelques critères: cris, couleur, comportement. Le lexique de l'avifaune, qui donne un exemple de la richesse lexicale et de l'unité de la langue malgache, a paru également dans le Bulletin des études africaines, Paris, 1987, vol. VII.

Nous signalons aussi l'existence d'une banque de données des terminologies malgaches au Centre d'information et de documentation scientifique et technique, à Tsimbazaza (Antananarivo).

B) Quels sont, en résumé, les travaux en cours et les résolutions du Séminaire d'information sur la terminologie?

La confrontation des expériences de divers pays en matière de terminologie amène les terminologues du séminaire aux résolutions et aux recommandations suivantes. On envisage:

1) la mise en place d'infrastructures, de divers organismes ou commissions, en vue de la coordination des activités terminologiques;

2) une normalisation des terminologies, en vue d'uniformiser la terminologie de chaque domaine, facilitant ainsi la communication entre les spécialistes de ce domaine: la terminologie sera unifiée, harmonisée dans le domaine de l'administration, de la santé publique, de la justice, du monde des sciences, de la technique et de la technologie;

3) une élaboration de dossiers préparatoires de normalisation terminologique en vue de permettre à un groupe (comité, commission) de rendre une décision ou de formuler une recommandation. Les dossiers consistent en une présentation du problème terminologique, en une étude du terme à normaliser, en une présentation de la documentation (ouvrages lexicographiques ou non) et d'une proposition, etc.;

4) la normalisation terminologique émanant d'un organisme dont l'autorité est reconnue; de plus, cette normalisation doit reposer non sur un choix arbitraire, mais sur un certain nombre de principes directeurs. Une planification et un dirigisme linguistique s'imposent. En effet, la normalisation terminologique doit être assortie de facteurs d'incitation ou de mesures contraignantes qui en assurent la mise en application.

Ainsi, le normalisateur voit dans la recommandation un moyen d'influer sur l'usage. C'est une façon d'informer et de sensibiliser le public de manière permanente. Par exemple, on recommande l'emploi d'un terme de préférence à d'autres synonymes et on cherche progressivement à éliminer les synonymes inutiles. On ne conservera qu'un ou deux termes dont l'un sera à caractère international si possible. Et c'est ce dernier terme qui sera normalisé;

5) une normalisation académique, sous l'autorité de l'Académie ou du ministère de l'Éducation;

6) la diffusion des terminologies normalisées par la voie ordinaire des moyens de communication (bulletin, publication des lexiques, etc.). Mais ce sont les utilisateurs cibles qui, finalement, établissent l'usage et sont susceptibles de dynamiser les termes normalisés;

7) la présentation du projet de Dictionnaire visuel thématique anglais-françaismalgache; une utilisation de l'écrit et des médias; l'établissement de banques de terminologies informatisées non seulement pour le stockage mais aussi pour la diffusion des données; la création de réseaux, avec l'aide technique et financière de l'OUA et de l'UNESCO. 
Cette série de recommandations, faites à partir du bilan des expériences et des résultats du travail terminologique à Madagascar, accorde donc une grande importance à la normalisation et à la diffusion, auprès de la masse, des terminologies normalisées.

Étant donné que l'utilisation de la langue nationale est destinée à permettre au peuple de connaître l'ensemble des sciences et des technologies autochtones et étrangères, l'effort du travail terminologique semble devoir porter essentiellement, non sur une malgachisation linguistique de la recherche fondamentale et de la haute technologie (ici, la langue internationale de communication et de travail reste le français), mais sur une malgachisation de l'enseignement des notions scientifiques et techniques de base.

\section{CONCLUSION}

Quelles sont donc les possibilités et les limites d'une malgachisation des terminologies scientifiques et techniques? La création d'une terminologie scientifique et technique en malgache répond essentiellement aux exigences d'un peuple qui veut acquérir des connaissances nouvelles dans une langue nationale. D'où le besoin d'une large diffusion et d'une vulgarisation des notions dans un malgache clair et accessible. La malgachisation semble vouloir revêtir ici un double aspect: un aspect pratique et un aspect d'identité nationale. Elle est surtout destinée à renforcer les capacités autochtones dans le domaine des sciences et des techniques de base; et la terminologie diffusée auprès de la masse joue un rôle primordial dans le transfert de la connaissance et de la technologie. Mais, pour avoir plus de chances de succès auprès du peuple, elle a besoin d'être adaptée, harmonisée et normalisée.

Or, si, selon une politique linguistique bien déterminée, la malgachisation sert de vulgarisation de masse en matière de science et de technologie, elle ne saurait prétendre concerner la langue de la recherche scientifique fondamentale et de la haute technologie. Ce sont là, pour ainsi dire, les limites de ses objectifs. En effet, à Madagascar, on fait toujours usage du français à l'université et dans les domaines de la géophysique, de la météorologie, de l'informatique, de la gestion et de la diplomatie. La langue de travail pour les conventions et les relations intemationales reste le français.

Et le fait même que ces terminologies élaborées en malgache soient encore inspirées du français par le biais et sur la base de la traduction, que la langue diplomatique reste le français, et que le français demeure la langue utilisée pour l'accès à la maîtrise de la haute technologie scientifique et économique montre, à Madagascar, l'articulation d'une langue nationale de spécialité et du français comme langue internationale de communication universitaire et scientifique.

Notes

1. Le professeur J. Dez, de I'Université Paris VII, l'a reformulé et en a fait une présentation critique sous le titre «Un catalogue ancien de noms de plantes malgaches» (1987, 55 p.) (Communication devant l'Académie malgache, 1988).

2. Librairie Orientale et Américaine, E. Guilmotto, éditeur, Paris (t. 1, 1908, t. 2, 1909), Émile Colin et Cie, Imprimerie de Lagy, E. Grévin, successeur.

3. Termes donnés dans le «lexique malagasy-malagasy-français», édité en 1986 par le CNAPMAD, ministère de l'Enseignement secondaire, Direction de la planification et de l'orientation de l'enseignement, Service de logistique pédagogique.

4. Dr. Razafindrazaka et Zoantsoa: Andram-panagasiana ny teny sy fiteny manokana momba ny rafi-batan' ny olombelona (litt. «Essai de malgachisation des termes spécialisés concernant le corps humain»).

5. E.M.F. Payne : «Basic syntactic structures in standard Malay», Kuala Lumpur, 1970.

6. Le transfert sémantique s'effectue, bien entendu, dans le passage du sens commun du terme au sens spécialise.

7. On observe ici encore pour les 3 exemples le phénomène de circumfixation. Ex. : préfixe FI- + radical ATY + suffixe -ANA. 
8. Le syntagme d'apposition, d'après J. Dez dans Syntaxe du malgache (Lille 1980), se definirait comme une juxtaposition, une association ou une apposition, sur le plan structural, de deux ou plusieurs éléments lexicaux pour former un syntagme, dont la signification nouvelle est la résultante de la signification de deux éléments pris séparément. Ainsi, masoandro «soleil» est un composé issu de maso «œil» et andro «jour». Dans notre étude des termes scientifiques malgaches, les syntagmes d'apposition, tels que sesibihy «nucléoplasme» (sesika «forme» et vihy «noyau»), ont pris des sens très spécialisés.

9. Il y a amalgame formel et sémantique des éléments de ces formations composées.

10. Concurremment à ces termes, nous connaissons d'autres traductions: gilaobola (emprunt), jaky (litt. «pourpre») «érythrocyte», kiady (litt. «défense») «leucocyte» (ces deux derniers termes sont donnés par le docteur Randrianasolo).

11. Calque de la définition française: matrice qui garde la même valeur si l'on opère une permutation de l'ordre de ses éléments ou de ses variables.

12. Ce sont des termes déjà existants et connus de la population locale, mais que les ingénieurs-géographes ont recueillis et rassemblés dans un lexique pour leur attribuer des signifiés techniques précis.

13. Cf. supra «La néologie morphologique».

14. «Remarques sur la malgachisation des termes scientifiques», BAM, 1976.

15. Celles de Martial Andrianaivo et du Service de logistique pédagogique, au ministère malgache de l'Enseignement secondaire et de l'Éducation de base, à Antananarivo.

16. Cf. supra «Introduction». Ces manuels sont conservés dans les archives de la Bibliothèque de l'Académie malgache à Tananarive.

17. Faravohitra et Ankadinandriana sont des quartiers de Tananarive.

18. «Remarques sur la malgachisation des termes scientifiques», BAM, t. 52/1-2, 1974 (1976), pp. 101-107; «Comment forger une langue scientifique malgache ?», BAM, t. 52/1-2, 1974 (1976) pp. 110-115.

19. E. Randrianasolo: op . cit. tome I, p. 44. L'auteur ne nous a pas donné d'exemples de mots malgaches «mal faits", mais on peut penser à un exemple contesté par Raoelina-Andriambololona (loc. cit.), que pourtant les usages scolaires semblent maintenir: celui de fifanampiana «addition», lequel, dans la langue usuelle, évoque plutôt l'idée morale d'entraide que d'ajout ou d'addition.

20. Sans doute pour maintenir le bilinguisme et pour faciliter la tâche des enseignants coopérants français et nationaux non formés dans la malgachisation, une décision ministérielle autorisait deux formes d'enseignement scientifique au choix, l'une entièrement en langue française, l'autre en langue malgache avec traduction française de toute terminologie, notamment en mathématiques.

21. Supra «Historique de la diffusion des terminologies».

22. Nous relevons ici des critiques soulevées dans divers séminaires à Madagascar et à Paris (par exemple, un séminaire sur la terminologie technique à l'INALCO le 16 novembre 1990, réunissant des étudiants et des enseignants linguistes).

23. «Inventaire des travaux en cours et des projets de terminologie», Réseau international de néologie et de terminologie, ACCT, Québec, Canada, 3 e trimestre 1988.

24. D'après des documents du STTTP (Objectifs et étapes du développement) rédigés par Assefa GabreMariam Tessema.

25. Parmi les pays représentés: Kenya, Tanzanie, Ouganda, Rwanda, Madagascar, Égypte, Israël, Pologne, Tchécoslovaquie, Ethiopie.

26. Nous pensons tout particulièrement à un ouvrage de référence comme Termes techniques français : essai d'orientation de la terminologie (établi par le Comité d'étude des termes techniques français. Actualités scientifiques et industrielles), Paris, Hermann éditeurs des sciences et des arts, 1972. La disposition d'un dictionnaire bilingue de termes techniques est conçue ainsi : terme proposé - identification du domaine définition - traduction proposée.

$\begin{array}{ll}\text { TRADUCTION EN MALGACHE DES TERMES USITÉS EN CARTOGRAPHIE } \\ \text { FANDIKANA HO MALAGASY IREO TENY FAMPIASA AO AMIN'NY SARINTANY } \\ \text { TERMES - TENY } & \text { DIKANI - TRADUCTION } \\ \text { 1. CARTE DE BASE } & \text { Sarintany fototra } \\ \text { 2. CARTE DÉRIVÉE } & \text { Zanatsarintany } \\ \text { 3. CARTOGRAPHIE } & \text { Taosarintany } \\ \text { 4. COURBE DE NIVEAU } & \text { Tsipika marindrano - Soritra mirahaabo } \\ \text { 5. COURBE INTERCALAIRE } & \text { Zana-tsipika marindrano - Zanatsoritra mirahaabo } \\ \text { 6. COURBE MAÎTRESSE } & \text { Renin-tsoritra mirahaabo } \\ \text { 7. COURBE SOUS-INTERCALAIRE } & \text { Zafintsoritra mirahaabo } \\ \text { 8. COURBE ISOTHERME } & \text { Soritra mirafana } \\ \text { 9. COURBE ISOHYETE } & \text { Soritra mirahale } \\ \text { 10. CREVE } & \text { Songon-tsarintany } \\ \text { 11. CROQUIS } & \text { Kisarisary }\end{array}$




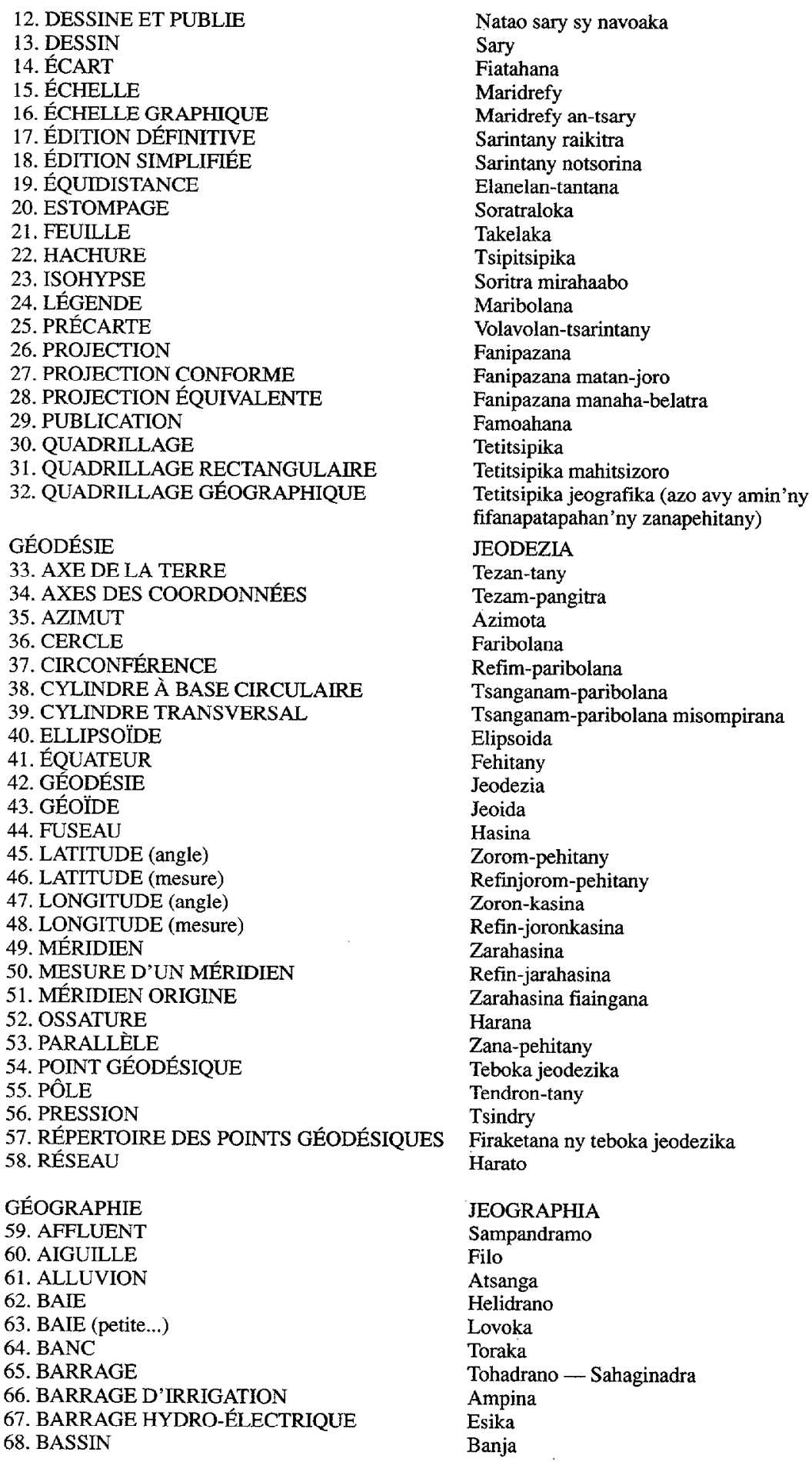




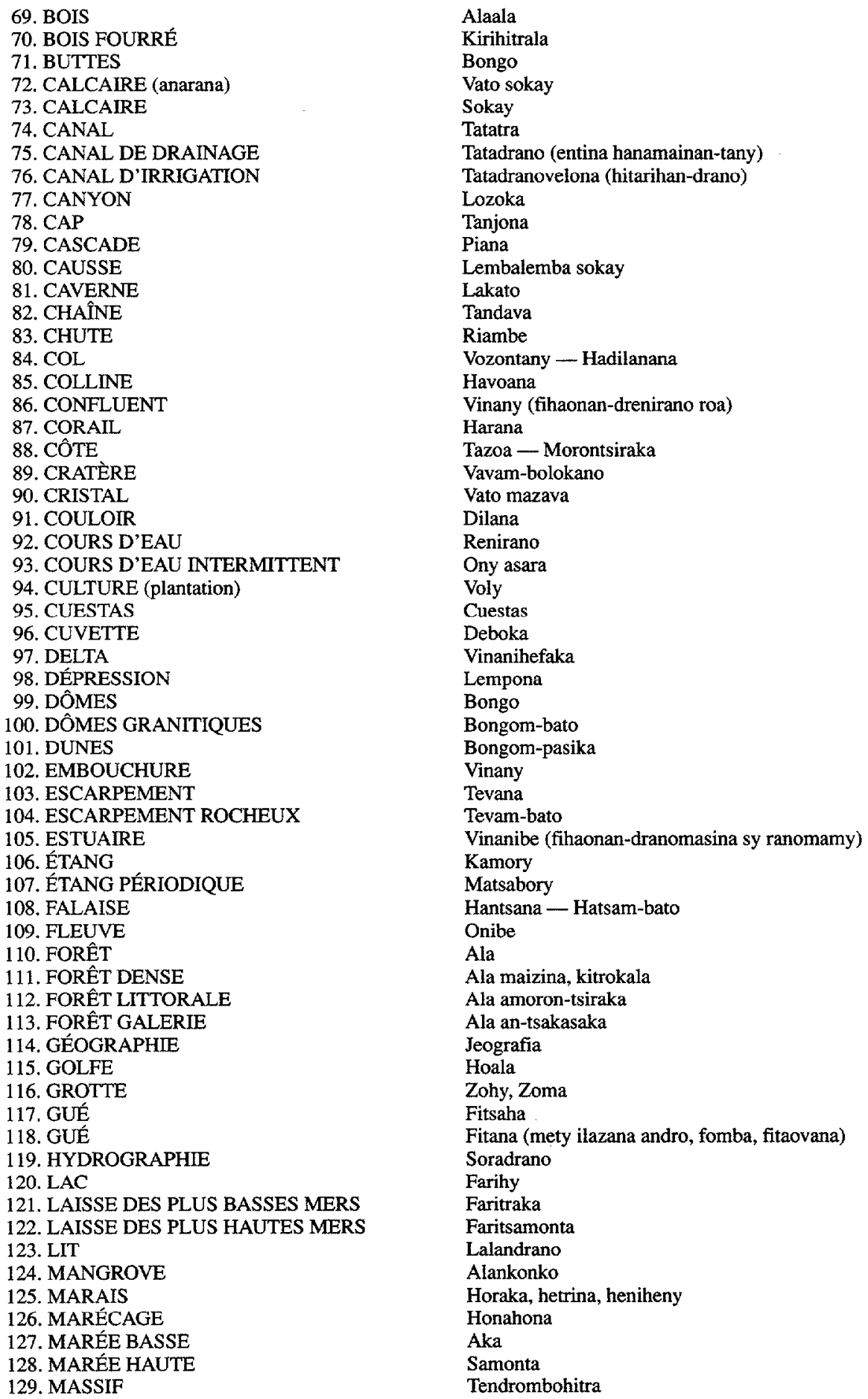


130. MONT

131. MONTAGNE

132. PALÉTUVIERS

133. PASSE

134. PÉNÉPLAINE

135. PIC

136. PLAINE

137. PLANTE

138. PLATEAU

139. PLATEFORME

140. POINTE

141. POINT CULMINANT

142. PUITS

143. QUARTZ

144. RADIER

145. RAPIDE

146. REBORD

147. RÉCIF

148. RELIEF

149. RELIEF (en...)

150. RIVIÈRE

151. ROCHE

152. ROCHER

153. RUISSEAU

154. SABLE

155. SABLES MOUILLÉS

156. SABLES SECS

157. SAISON DE PLUIE

158. SAISON SĖCHE

159. SAVANE

160. SAVANE ARBORÉE

161. SAVANE ARBUSTIVE

162. SEUIL

163. SOMMET

164. STEPPEFatrana

165. TALUS

166. THALWEG

167. TORRENT

168. TOURBES

169. TOURBILLON

170. TOURBILLON

171. VALLÉE

172. VASE

173. VÉGÉTATION NATURELLE

174. VÉGÉTATION DE TYPE HUMIDE

175. VÉGÉTATION DE TYPE SEC

176. VERSANT

PHOTOGRAMMÉTRIE ET

PHOTOGRAPHIES AÉRIENNES

177. AÉROCHEMINEMENT

178. APPAREIL DE RESTITUTION

179. BASCULEMENT

180. MOSAÏOUE

181. COUVERTURE PHOTOGRAPHIQUE

182. COUVERTURE PHOTOGRAPHIOUE AÉRIENNE

183. PHOTOGRAPHIE AÉRIENNE

184. PHOTOGRAMMÉTRIE

185. PHOTO REDRESSÉE

186. PRISES DE VUES AÉRIENNES
Tendrombohitra kely

Tendrombohitra

Honko

Ararano

Sarilemaka

Kitsotendro

Lemaka

Zavamaniry

Lembalemba

Hamarenana

Lohatanjona

Tendro

Vovo

Vato velona

Ranofotsy

Rano mare

Kiha

Harandriaka

Vohontany

Mivohitra

Ony

Vato (Roche Volture)

Harambato

Sakelidrano - Sakasaka

Dilatra (fotaka eny amoron-drano)

Fasi-dena

Fasi-maina

Asara

Asotry

Hivoka

Roranga

Akata

Hoatra - Tokonana

Tampo

Solampy

Lahidrano

Riaka

Fompotra

Ranomody (rano)

Tadio (rivotra)

Lohasaha

Fotadrevo, dilatra

Volinanahary

Zavamaniry amin'ny faritra be orana

Zavamaniry amin'ny faritra kely orana

Tehezana

FOTOGRAMETRIA SY SARY

TAM-PIARAMA-NIDINA

Tanjozodrefy

Famadihantsary (raha soratana teny roa dia milaza ny asa atao)

Fampihilanana (fitaovam-petratsary)

Tambatambatsary

Rako-tsary

Rako-tsary tam-piaramanidina

Sary tam-piaramanidina - Sary an-habakabaka

Fotogrametria

Sary voaarina

Fangalan-tsary an-habakabaka 


\author{
187. REDRESSEMENT \\ 188. RESTITUTION \\ 189. STÉRÉOMINUTE \\ 190. STÉRÉOPRÉPARATION \\ 191. STÉRÉOSCOPE \\ 192. STÉRÉOSCOPIE \\ TERMES GÉNÉRAUX \\ 193. AÉRODROME \\ 194. AÉROPORT \\ 195. AÉROGARE \\ 196. BAC \\ 197. CÂBLE TRANSPORTEUR \\ D'ÉNERGIE ÉLECTRIQUE \\ 198. CALVAIRE \\ 199. CAMPEMENT \\ 200. CANAL DE MOZAMBIQUE \\ 201. CHÂTEAU D'EAU \\ 202. CHEF-LIEU \\ 203. CHEMIN DE FER \\ 204. CHEMIN D'EXPLOITATION \\ 205. CENTESIMAL \\ 206. CIMETIËRE \\ 207. CITERNE \\ 208. DIGUETTE \\ 209. DISPENSAIRE \\ 210. ÉGLISE \\ 211. ÉNERGIE ÉLECTRIQUE \\ 212. GARE (station) \\ 213. GARGOTE \\ 214. GÎTE D'ÉTAPE \\ 215. GROTTE \\ 216. HÔPITAL \\ 217. HÔTEL \\ 218. JETÉE \\ 219. LIGNE TÉLÉGRAPHIQUE \\ 220. LIMITE \\ 221. LIMITE ADMINISTRATIVE \\ 222. MARCHE \\ 223. MOSQUÉE \\ 224. PASSAGE À NIVEAU \\ 225. PASSAGE INFÉRIEUR \\ 226. PASSAGE SUPÉRIEUR \\ 227. PHARE \\ 228. PILE \\ 229. POSTE MÉDICAL \\ 230. RÉSERVOIR \\ 231. RESTAURANT \\ 232. RIZIÈRE \\ 233. RIZIËRE DE TANETY \\ 234. ROUTE NATIONALE REVÊTUE \\ 235. ROUTE NATIONALE SAISONNIËRE \\ 236. ROUTE NATIONALE PERMANENTE \\ 237. ROUTE CARROSSABLE \\ 238. ROUTE BORDÉE D'ARBRES \\ 239. ROUTE PRATICABLE UNE PARTIE \\ DE L'ANNÉE \\ 240. ROUTE EN REMBLAIArabe tovontany \\ 241. ROUTE EN CONSTRUCTION \\ 242. RUINES \\ 243. SECONDE \\ 244. SENTIER
}

Fanarenana (sary)

Petratsary - Vaditsary (vadika sary)

Matoam-petratsary

Omana fivohitsary

Famohijery (famohitra jery)

Fivohijery (fivohitra jery)

Fitodiam-piaramanidina

Seranam-piaramanidina

Fitobiam-piaramanidina

Zahatra

Mahazaka angovom-baratra

Tanam-bokovoko

Toby

Ranomasin'i Mozambika

Siniben-drano

Voënana

Lalamby

Lalam-pitrandrahana

Ara-pototra zato

Vondrom-pasana

Sinibe

Tahalaka - Valam-parihy

Famoaham-panafody

Fiangonana Katolika

Angovom-baratra

Gara

Hotely kely

Tranom-bahiny

Zony

Hopitaly

Hotely (misy fandriana)

Taribin'ny telegrafy sy telefaonina

Fangitra

Fangitra fizaram-paritany

Tsena

Fiangonana Silamo

Hitsa-dalamby

Dikain-dalamby

Dikan-dalamby

Jiron-dranomasina

Vatonaratra

Tobim-pahasalamana

Tobin-drano

Hotely be

Tanimbary, Hosy

Kipany

Arabem-pirenena voarakotra tara

Arabem-pirenena azo aleha amin'ny maintany

Arabem-pirenena azo aleha mandavan-taona

Arabe azon'ny fiara aleha

Arabe tomefy hazo

Arabe azon'ny fiara aleha amin'ny maintany

Arabe vao hovakiana

Tanàna haolo

Segondra

Lalan-tongotra 
245. SEXAGÉSIMAL

246. STATION (arrêt)

247. TEMPLE

248. TOMBEAU

249. TOUR

250. TUNNEL

251. VILLAGE

252. VOIE MÉTRIQUE - VOIE ÉTROITE

253. VOLCAN

TOPOGRAPHIE

254. AIRE

255. ALIDADE

256. ALIDADE NIVELATRICE

257. ALTIMÉTRIE

258. ALTIMÉTRIQUE

259. ALTTTUDE

260. ANGLE HORIZONTAL

261. ANGLE NADIRAL

262. ANGLE VERTICAL

263. ANGLE ZÉNITHAL

264. BASE

265. BORNE

266. CERCLE DROIT

267. CERCLE GAUCHE

268. CHEMINER

269. CHEMINEMENT

270. CLISIMĖTRE

271. COMPENSATION

272. COMPLÈTEMENT

273. COMPLÈTEMENT SUR LE TERRAIN

274. COMPAS DE CENTRAGE

275. COMPAS DE STATION

276. COORDONNÉES

277. CỐTE

278. CÔTE DE SOMMET

279. CÔTE DE FOND

280. CÔTE LOCALE

281. COURBES DE NIVEAU, ISOHYPSE

282. COURBES BATHYMÉTRIQUES

283. CRAPAUD DE NIVELLEMENT

284. CROQUIS (Tachéométrie)

285. DÉCLINER

286. DÉCLINATOIRE

287. DEGRÉ

288. DÉTAIL (en...)

289. DÉTERMINER

290. DÉTERMINATION

291. DISTANCE

292. DISTANCE HORIZONTALE

293. DISTANCE VERTICALE

294. DISTANCE SUIVANT LA PENTE

295. DISTANCEMETRE

296. DOUBLE PAS

297. ÉCHELLE

298. ÉQUERRE OPTIQUE

299. ÉQUIDISTANCE

300. FAILLE

301. FAIRE UNE VISÉE

302. FERMETURE

303. FERMER UN TOUR D'HORIZON

304. FERMETURE D'ANGLE
Ara-pototra enimpolo

Fiatoana (halte)

Fiangonana Protestanta

Fasana

Tatao (raha ampiasaina)

Tonelina

Tanàna

Lalamby 1 metatra - Latsaky ny 1 metatra

Volokano

TOPOGRAFIA

Velatra

Alidady

Fandajahaambo

Fandrefihaambo

Ara-drefihaambo

Haavo - Haambo

Zoro marindrano

Zoro jadona

Zoro jidina

Zoro traka

Fotodrefy

Baorina

Faribolana havanana

Faribolana havia

Mitaridrefy

Taridrefy

Mpanjoro firaika

Fanoneran-drefy

Famenoana

Famenoana teny an-toerana

Kômpa fampijoroana

Kômpa fanivoana

Fangitra

Refina haavo - Refinkaambo

Refin-kamboasana

Refin-kaiba

Refinkaambo foronina

Soritra mirahaabo

Soritra mirahalaly

Akalan-danjahaambo

Vangovangon-tsary

Mamaritra avaratra

Mpamaritra avaratra

Degre

Antsipiria

Mamaritra

Famaritana

Zeva - Halavirana

Zeva - Halavirana marindrano

Zeva - Halavirana jidina

Zeva miraika

Fandrefy zeva elektronika

Dingandroa

Maridrefy

Sokera faenty

Elanelan-tantana

Tsefaka

Manao tily mivantana

Hidy

Manidy refy an-kodina

Elana hidin-joro 


\author{
305. FERMETURE DE DISTANCE \\ 306. FERMETURE D'ALTITUDE \\ 307. FIL À PLOMB \\ 308. FIL INTÉRIEUR \\ 309. FIL SUPÉRIEUR \\ 310. FIL DE RÉTICULE \\ 311. FIN DE POINTAGE (vis...) \\ 312. GRADE \\ 313. HAUTEUR \\ 314. HORIZONTAL \\ 315. INTERSECTER \\ 316. INTERSECTER UN POINT \\ 317. INTERSECTION \\ 318. LECTURE \\ 319. LECTURE AVANT \\ 320. LECTURE ARRIÈRE \\ 321. LIMBE (cercle gradué) \\ 322. LUNETTE \\ 323. MESURE DE DISTANCE \\ 324. METTRE EN STATION \\ 325. METTRE AU POINT (dessin) \\ 326. MISE EN STATION \\ 327. MINUTE DE LEVÉ \\ 328. NIVEAU \\ 329. NIVEAU - NIVELLE \\ 330. NIVEAU À LUNETTE \\ 331. NIVELLEMENT \\ 332. NIVELLE EN «T» \\ 333. OBSERVATION \\ 334. OBSERVER \\ 335. OROGRAPHIE \\ 336. OUVERTURE \\ 337. OUVRIR UN TOUR D'HORIZON \\ 338. PENTE \\ 339. PIED DE PLANCHETTE \\ 340. PIC \\ 341. POINT COTE \\ 342. POINT DE STATION \\ 343. PORTÉE \\ 344. COURTE PORTÉE \\ 345. MOYENNE PORTÉE \\ 346. LONGUE PORTÉE \\ 347. PRECISION DE MESURE \\ 348. PRISME À RÉFLEXION \\ 349. PLAN \\ 350. PLANCHETTE \\ 351. PLANIMÉTRIE \\ 352. PLANIMÉTRIQUE \\ 353. RATTACHER \\ 354. RATTACHEMENT \\ 355. RAYONNER \\ 356. RAYONNEMENT \\ 357. RECOUPER \\ 358. RECOUPEMENT \\ 359. REDRESSER \\ 360. REDRESSEMENT \\ 361. RELEEVEMENT \\ 362. RELIEF \\ 363. REMBLAI \\ 364. REPÈRE \\ 365. REPĖRE DE NIVELLEMENT
}

Elana hidin-jeva

Elana hidin-kaambo

Tadin-jidina

Folim-panjeva ambany

Folim-panjeva ambony

Folim-panjeva

Visy fampifanojoana farany

Grady

Haavo

Marindrano

Mampifanapaka tily

Mampifanapaka tily eo amin'ny teboka

Fifanapahan-tily

Vaky

Vaky roso

Vaky todika

Faribolana voateti-drefy

Faenty

Fandrefesan-jeva - Fanjevana mivan-tana

Mampijoro

Manadio ho raikitra

Fampijoroana fitaovam-pandrefesana

Matoan-drefitany

aabo

Levina

Levina am-paenty

Lanja haambo

Levina " $T$ "

Fitiliana

Mitily

Soribohitany

Fanokafana

Manokatra refy an-kodina

Kisolosolo

Tongom-pafana

Lingilingy

Teboka voarefy haambo, tebo-kaambo

Teboka fampijoroana

Toraka

Mariy toraka

Antonon-toraka

Lavi-toraka

Fanamarinan-drefy

Prisma fampoliana

Planina

Fafana

Planimetria

Planimetrika

Mandrohy

Fandrohizana

Mampisandrahaka tily

Fampisandrahan-tily

Mampifandray tily

Fampifandraisan-tily

Manarina

Fanarenana

Fanarenan-tily - Fifanaparitahan-tily mifototra

Fivohitany

Tototany

Marika

Marindanja haambo 
366. SITOMÈTRE

367. STATION CENTRÉE (teboka)

368. STATION EXCENTRÉE (teboka)

369. SURFACE

370. SURFACE DU CERCLE

371. TALUS

372. THÉODOLITE

373. TOPOGRAPHIE

374. TOUR

375. TRIANGULATION

376. TRIANGULATION COMPLÉMENTAIRE

377. TRILATÉRATION

378. VERTICAL

379. VIS DE POINTAGE

380. VIS DE FIN DE POINTAGE

381. VISÉE ASCENDANTE

382. VISÉE DIRECTE

383. VISÉE INDIRECTE

384. VISÉE
Mpanjoro firaika

Fampijoroana voamarika

Fampijoroana miataka

Velatra

Vela-paribolana

Solampy

Teodolita

Topografia

Tongoambato

Fiteloanjoro - Fiteloanjoro misesy

Fiteloanjoro famenony

Fiteloandafy

Jidina

Visy fampifanojoana

Visy fampifanojoana

Tily miakatra

Tily mivantana

Tily mifototra - an-kolaka

Tily - Hitsy tily — Kendry 